\title{
Strain distribution on material surfaces during combustion regime transitions
}

\author{
F. Hampp, R. P. Lindstedt* \\ Department of Mechanical Engineering, Imperial College, London, SW7 2AZ, UK
}

\begin{abstract}
A multi-fluid state approach is used to analyse the underlying conditions for burning mode transitions from close to the corrugated flamelet regime to distributed reactions. Turbulent $\left(\mathrm{Re}_{t} \simeq 380\right)$ premixed DME/air flames were aerodynamically stabilised in a back-to-burnt opposed jet configuration with the Damköhler number varied through the mixture stoichiometry. Simultaneous Mie scattering, OH-PLIF and PIV allowed the delineation of five separate fluid states (reactants, combustion products, mixing fluid, mildly and strongly reacting fluids) with associated material surfaces. The analysis shows self-sustained flames in low strain regions with a collocated flow acceleration for higher Damköhler numbers. By contrast, in highly strained regions (e.g. beyond the twin flame extinction point) the burning mode is governed by the counter-flowing hot combustion products resulting in increased levels of vorticity and an absence of a preferential dilatation direction. The current analysis provides novel insights into combustion regime transitions by means of (i) strain rate statistics conditioned upon material surfaces and (ii) the evolution of fluid state interface probabilities as a function of the Damköhler number. The work further shows (iii) that the combustion mode influences scalar transport and that increased levels of turbulence retards the transition to non-gradient transport.
\end{abstract}

Keywords: Strain rate, Combustion regime transition, Premixed DME Flames, Multi-Fluid Statistics, Fractal Grid Generated Turbulence

\footnotetext{
${ }^{*}$ Corresponding author. Fax: +44 2075945696.

Email address: p.lindstedt@imperial.ac.uk (R. P. Lindstedt)
} 


\section{Introduction}

The study of turbulence-chemistry interactions in different combustion regimes presents a formidable challenge that favoures the use of canonical burner configurations to advance fundamental understanding. Examples include the Sandia - Sydney piloted jet flames $[1,2]$ and the opposed jet configuration $[3,4]$. The reaction zone broadening at low Damköhler (Da) numbers promotes reduced emissions by removing sharp reaction fronts $[5,6]$, but frequently requires an enthalpy source to avoid flame extinction [7]. Mastorakos et al. [8] and Goh et al. [9, 10] used a back-to-burnt (BTB) opposed jet configuration to stabilise ultra lean flames beyond the extinction point. Hampp and Lindstedt [11] used a BTB configuration and a multi-fluid approach to quantify combustion regime transitions of premixed methane flames from close to the corrugated flamelet regime to distributed reaction zones with $0.06<\mathrm{Da}<1.6$ at a constant turbulent Reynolds numbers $\left(\operatorname{Re}_{t} \simeq 380\right)$. Coriton et al. [12] investigated the impact of strain on turbulent premixed methane flames, stabilised by stoichiometric combustion products, in a BTB opposed jet configuration. Local and intermittent flame extinction was observed for bulk strain rates that exceeded the extinction conditions of laminar counterflow flames and Böhm et al. [13] identified the strain rate as the driving measure for flame extinction. Hartung et al. [14] studied experimentally the impact of turbulence intensity and showed the effects of dilatation induced strain on the principal components at $\mathrm{Da}>1.0$. A preferential alignment of strain with the scalar gradient was found experimentally by Steinberg et al. $[15,16,17]$ for Da $>1$ and $150<\mathrm{Re}_{t}<500$. The impact of the Da on the preferential strain alignment with the flame surface normal was investigated by Chakraborty and Swaminathan [18] for flames in the thin reaction zone and corrugated flamelet regimes via direct numerical simulation $(\mathrm{DNS})$ with $\mathrm{Re}_{t} \simeq 50$. The impact of dilatation was found to dominate turbulent strain effects at high Da causing a preferential alignment of the extensive component with the flame normal and consequently scalar gradient dissipation. The opposite trend was noted for low Da. The trends were verified by DNS studies in the range of $0.2<\mathrm{Da}<7$ at $\operatorname{Re}_{t} \leq 110[19,20]$.

The multi-fluid concept of Spalding [21] was evaluated by Hampp [22] as a possible 


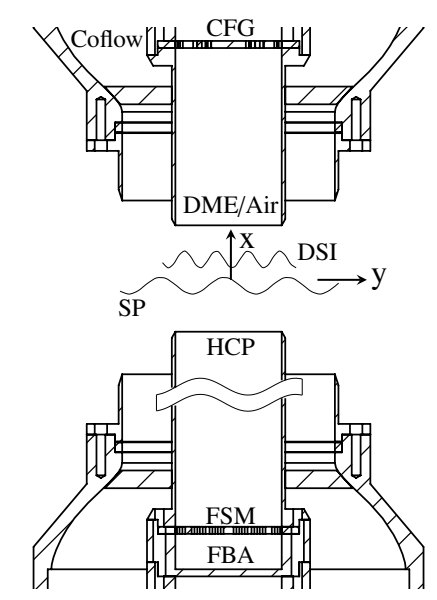

Figure 1: Schematic of the experimental configuration with the coordinate system convention indicated. SP - stagnation plane; DSI - density segregation iso-contour; HCP - hot combustion products; CFG - cross fractal grid; FSM - flame stabilising mesh; FBA - flash back arrestor.

extension to bimodal (two-fluid) approaches [23] via quantification of intermediate fluid states. The current work provides insight into the underlying causes of burning mode transitions from close to the corrugated flamelet regime to distributed zones by means of (i) strain rate statistics conditioned upon material surfaces and (ii) the evolution of fluid state interface probabilities as a function of the Damköhler number. The work further shows (iii) that the combustion mode influences scalar transport and that increased levels of turbulence retards the transition to non-gradient transport. The fuel selected (DME) is a Diesel replacement [24] with a comparatively well established chemistry [25].

\section{Experimental Setup}

The current opposed jet geometry (c.f. Geyer et al. [3]) is schematically depicted in Fig. 1. A variant was previously used by Goh et al. $[4,9,26,27]$ with the current configuration identical to that of Hampp et al. [11, 22]. The burner was operated in a BTB mode with premixed reactants injected through the upper nozzle (UN) and stabilised against hot combustion products (HCP) emerging the lower nozzle (LN). The BTB configuration provides individual control of chemical and turbulence timescales [10, 22], accurate boundary conditions [4], excellent optical access [3, 28] and aerodynamic flame stabilisation [26]. The nozzle separation was set to $1 \mathrm{D}(\mathrm{D}=30 \mathrm{~mm})$. 
Table 1: Overview of experimental conditions. The extinction strain rates $a_{q}\left(s^{-1}\right)$ were computed in the corresponding twin flame configuration [22]. The Euclidean norm $\left(|a|=\left(a_{n}^{2}+a_{t}^{2}\right)^{1 / 2}\right)$ was evaluated along the reactant $(\mathrm{R})$ and strongly reacting (SR) fluid surfaces. The integral length scale $\left(L_{I}=4.1 \mathrm{~mm}\right)$ and velocity fluctuation $\left(\mathrm{u}^{\prime}=1.6 \pm 0.04 \mathrm{~m} \mathrm{~s}^{-1}\right)$ were kept constant.

\begin{tabular}{l|ccccc}
\hline$\Phi$ & 0.2 & 0.4 & 0.6 & 0.8 & 1.0 \\
\hline $\mathrm{u}^{\prime} / \mathrm{S}_{L}$ & 39.8 & 26.5 & 7.57 & 4.08 & 3.18 \\
$\mathrm{~L}_{I} / \delta_{f}$ & 3.15 & 3.76 & 8.91 & 15.2 & 17.1 \\
$\mathrm{Re}_{\mathrm{t}}$ & \multicolumn{5}{|c}{$386 \pm 15$} \\
$\mathrm{Da}$ & 0.08 & 0.14 & 1.2 & 3.9 & 5.1 \\
$a_{q} \mathrm{~s}^{-1}$ & - & - & 600 & 2000 & 3100 \\
$|a|_{R} \mathrm{~s}^{-1}$ & 2669 & 2627 & 2355 & 1761 & 1603 \\
$a_{q} /|a|_{S R}$ & - & - & 0.58 & 1.8 & 3.1 \\
\hline
\end{tabular}

\subsection{Upper Nozzle Flow Conditions}

Premixed DME/air mixtures with varying equivalence ratios $(\Phi=0.2-1.0)$, a constant initial temperature $\left(T_{r}=320 \mathrm{~K}\right)$ and bulk velocity $\left(U_{b}=11 \mathrm{~m} \mathrm{~s}^{-1}\right)$, were injected through the UN. A cross fractal grid (CFG) [4] was installed $50 \mathrm{~mm}$ upstream of the UN exit to provide a multi-scale [26] turbulent flow with $\operatorname{Re}_{t}=L_{I} \cdot u^{\prime} / \nu_{r} \simeq 380 \mathrm{in}$ the absence of bulk flow instabilities [4]. The integral length scale of turbulence $\left(L_{I}=\right.$ $4.1 \mathrm{~mm}$ ) was inferred from hot wire anemometry with the average velocity fluctuation $\left(u^{\prime}=1.6 \pm 0.04 \mathrm{~ms}^{-1}\right)$ measured using PIV. The Da $\left(=\left(L_{I} \cdot S_{L}\right) /\left(\delta_{f} \cdot u^{\prime}\right)\right)$ range was estimated to $0.08<\mathrm{Da}<5.1$ based on the $5-95 \%$ fuel consumption thickness $\left(\delta_{f}\right)$ and the laminar burning velocity $\left(S_{L}\right)$ [22], with details provided in Table 1 . The values were inferred from strained laminar BTB opposed jet calculations [29] that also provided the kinematic viscosity $\left(\nu_{r}\right)$ in the reactants. The relation of Kostiuk et al. [30] was used to estimate the turbulent $\left(a_{I}=\left(\varepsilon_{r} / \nu_{r}\right)^{1 / 2} \simeq 3200 \mathrm{~s}^{-1}\right)$ and bulk $\left(a_{b}\right.$ $\left.=2 \mathrm{U}_{b} / \mathrm{H} \simeq 750 \mathrm{~s}^{-1}\right)$ strain, where $\varepsilon_{r}$ is the rate of dissipation in the reactants [31]. The Kolmogorov length scale $\left(L_{\eta}=\left(\nu_{r}^{3} / \varepsilon_{r}\right)^{1 / 4}\right)$ was estimated to $73 \pm 1.6 \mu \mathrm{m}$.

\subsection{Lower Nozzle Flow Conditions}

The stagnation plane was positioned close to the burner centre by momentum matching with $U_{b}=4.3 \mathrm{~m} \mathrm{~s}^{-1}$ at STP conditions [22]. Premixed $\mathrm{H}_{2} /$ air $(\Phi=1.0)$ mixtures were used for stable flame anchoring on the FSM (see Fig. 1) and to provide an enthalpy source with close to fully combusted HCP [32]. The reactants were diluted with $22 \% \mathrm{CO}_{2}$ by volume to reduce the temperature $\left(T_{H C P}=1700 \mathrm{~K}\right)$. 


\subsection{Measurement Setup}

Simultaneous Mie scattering, particle image velocimetry (PIV) and hydroxyl-planar laser induced fluorescence (OH-PLIF) measurements were performed using the technique of Kerl et al. [33]. The OH-PLIF (281.7 nm) and PIV (532 nm) laser light sheets had a height of $1 \mathrm{D}$, thicknesses $<250 \mu \mathrm{m}$ and $<500 \mu \mathrm{m}$, respectively, and pulse energies around $2 \mathrm{~mJ}$ and $30 \mathrm{~mJ}$ [22]. For statistical accuracy of relatively rare events, 3000 instantaneous and statistically independent $(5 \mathrm{~Hz})$ image pairs were recorded for each condition (i.e. $\Phi$ ). The air streams were seeded separately with aluminium oxide particles with $d_{p, 90}<1.66 \mu \mathrm{m}$ corresponding to a Stokes number $\left(=\tau_{p} / \tau_{\eta}\right)<1$. The Kolmogorov timescale in the reactants $\left(\tau_{\eta}=\left(\nu_{r} / \varepsilon_{r}\right)^{1 / 2}\right)$ was estimated to $310 \mu \mathrm{s}$. The particle relaxation times $\left(\tau_{p}\right)[34]$ were $\tau_{p} \approx 30 \mu \mathrm{s}$ and $10 \mu \mathrm{s}$ with the smallest PIV timescales [35] estimated to $65 \mu \mathrm{s}$ and $30 \mu \mathrm{s}$ for the UN reactants and the LN HCP flow. Cross-correlation PIV with adaptive interrogation regions of decreasing size (128 $\times 128$ to $48 \times 48$ with $75 \%$ overlap) was performed to incorporate the local flow field acceleration, providing a spatial resolution and vector spacing of $\lambda_{P I V}=300 \mu \mathrm{m}$ [36]. The spatial resolution $\left(4 \cdot L_{\eta}\right.$ versus $\left.2-5 \cdot L_{\eta}[15,37,38,39]\right)$ and laser sheet thickness $\left(3.4 \cdot L_{\eta}\right.$ versus $\left.2.5 \cdot L_{\eta}[39]\right)$ suggest that most (in-plane) fine scale structures of the turbulent flow are resolved. For out-of-plane contributions, Nishino et al. [40] showed good agreement between the circumferential and radial velocity fluctuation in stagnating flows. The maximum radial fluctuations were determined to $\sim 0.19 \mathrm{U}_{b}[22]$, which corresponds to an out-of-plane displacement [40] of the order of $50 \mu \mathrm{m}$ (i.e. $<$ $\left.L_{\eta}\right)$. The instantaneous planar rates of strain are - to some degree - underestimated due to $3 \mathrm{D}$ effects. However, the error due to the out-of-plane movement contributing to the in-plane particle displacement is estimated to $<7.5 \mu \mathrm{m}$ (i.e. $\sim 0.1 \cdot L_{\eta}$ ) [41].

\subsection{Multi-Fluid Post-Processing}

The stabilisation of reactants against an external enthalpy source removes conventional twin flame extinction criteria [8] and ultra lean flames with $\mathrm{Da}<1$ can be sustained. This suggests the differentiation of self-sustained (flamelet-like) burning from supported/distributed chemical activity (e.g. auto-ignition related oxidation). A 
multi-fluid (MF) analysis [22] can accommodate the identification of intermediate fluid states, defined below, in addition to reactants and products.

Reactant Fluid: PIV tracer particle based density segregation (DS) techniques are widely used, e.g. $[26,42,43]$, to detect the first thermal alteration of a flow. The present algorithm is a variant that is capable of detecting multiple and fragmented splines [22] and is used to identify the reactants (R). The UN (LN) stream features a high (low) particle seeding density to provide an unambiguous detection of the density segregation iso-contour (DSI) due to turbulent mixing or exothermic reactions.

Mixing Fluid: The turbulent transport of HCP across the stagnation plane into cold reactants results in a thermally altered fluid state with a quenched $\mathrm{OH}$ signal. The detectable change in seeding density can be attributed to mixing (M).

Product Fluid: The $\mathrm{OH}$ arising from DME combustion products and the HCP equilibrium concentration are combined to define the product fluid $(\mathrm{P})$.

Strongly Reacting Fluid: Self-sustained flames detach from the stagnation plane and become independent of the HCP. Accordingly, conventional aerothermochemical conditions apply. Hence, the mixture specific twin flame extinction strain rate (see $a_{q}$ in Table 1) provides a reference point with the corresponding $\mathrm{OH}$ concentrations used to segregate strongly reacting (SR) self-sustained flames.

Mildly Reacting Fluid: Regions with modest $\mathrm{OH}$ signal levels can stem from (i) ignition events, (ii) the decay of $\mathrm{OH}$ concentration in combustion products or (iii) chemically active material that is diluted and sustained by the HCP. Such regions are not further delineated and are denoted as mildly reacting (MR).

The constant $\mathrm{OH}$ signal in the $\mathrm{HCP}$ is used as the reference point for intensity bands and relates the measured $\mathrm{OH}$ signal to theoretically determined concentrations.

\subsubsection{Spatial Multi-Fluid Resolution}

The MF interrogation regions $(-11.6<\mathrm{x}<14.1$ and $-15.0<\mathrm{y}<15.0 \mathrm{~mm})$ are resolved by $708 \times 829$ pixels. The spatial accuracy of the MF analysis is governed by the required smoothing of the Mie images and was estimated to $\lambda_{M F}=250 \mu \mathrm{m}$ via an USAF target [44]. This is of the order of the thinnest laminar flame thickness $\left(\delta_{f}=\right.$ $222 \mu \mathrm{m}), 3 \cdot L_{\eta}$ and the PIV resolution. The mean scalar dissipation layer thickness [45] 


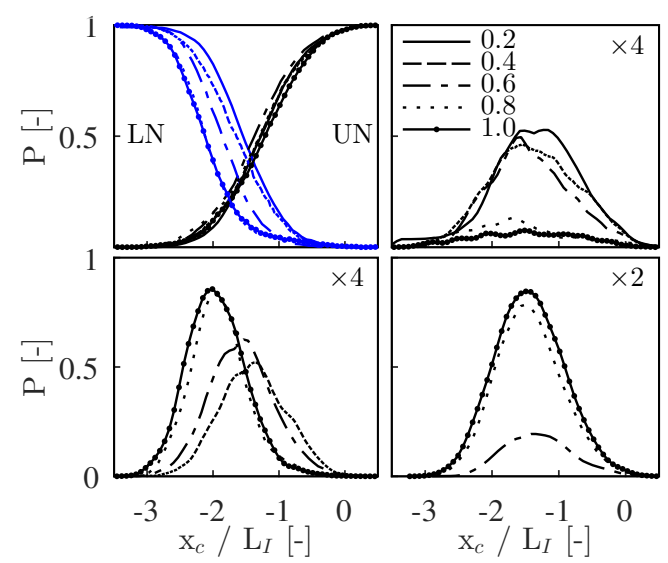

Figure 2: Multi-fluid probability statistics in physical space along the stagnation point streamline and aligned at the $\bar{c}=0.02$ iso-contour. Top left: Reactant (black) and product fluid probability (blue/grey); Right: Mixing fluid probability $\times 4$; Bottom left: Mildly reacting fluid probability $\times 4$; Right: Strongly reacting fluid probability $\times 2$. Legend entries refer to the equivalence ratio with the corresponding Da listed in Table 1.

$\lambda_{D} \approx 11.2 \cdot L_{I} R e_{t}^{-3 / 4} S c^{-1 / 2}=621 \pm 18 \mu \mathrm{m}$ is resolved. The Batchelor scale [46] in the $\mathrm{HCP}\left(\lambda_{B} \simeq 370 \mu \mathrm{m}\right)$ is of the order of the spatial resolution and under-resolved in the reactants $\left(\lambda_{B}=86 \pm 1.5 \mu \mathrm{m}\right)$.

\section{Results and Discussion}

Before exploring the strain distribution along the selected material surfaces, the evolution of the fluid states as a function of the Da number is discussed to illustrate burning mode transitions. The investigated range of $u^{\prime} / S_{L}$ and $L_{I} / \delta_{f}$ for $\Phi=0.2-$ 1.0 is listed in Table 1. In terms of a classical Borghi diagram [47], conditions from corrugated flamelets to distributed reactions are covered. The analyses in Sections 3.1 and 3.3 were conducted along the theoretical stagnation point streamline (SPS with $\mathrm{y}=0$ in Fig. 1). The results in Section 3.2 include the movement of the stagnation point [28] within the radial limit of $\pm 0.5 L_{I}$ away from the SPS.

\subsection{Multi-Fluid Flow Analysis}

The reactant fluid indicates a flame brush thickness $(5-95 \%)$ limited to around $2 L_{I}$ by turbulent transport that is only modestly affected by the Da number, see Fig. 2 . Profiles are aligned on a derived (see Section 3.3) bimodal [26, 42, 43] reaction progress variable iso-contour $(\bar{c}=0.02[48])$ showing a shift of the product fluid away from the 
reaction onset $\left(\mathrm{x}_{c}=0\right)$ with increasing Da due to enhanced dilatation. In physical space, the reaction onset shifts upstream with increasing Da due to the increased burning velocity, see Section 3.3. The chemistry impact is highlighted by the intermediate fluid states with the mixing fluid being favoured at low Da. The mildly reacting fluid probability increases and shifts away from the origin with increasing Da number. A distinct augmentation of the strongly reacting fluid is evident for Da $>1.0$.

The above findings motivated a statistical analysis of fluid state interfaces encountered by traversing through the quinary multi-fluid field along the SPS as shown in Fig. 3. No restrictions are imposed on the multi-fluid topology. Fluid pockets thinner than the multi-fluid resolution $(250 \mu \mathrm{m})$ were omitted as shown in Fig. 3, where the mixing to product $(\mathrm{M}-\mathrm{P})$ fluid interface was omitted (green layer $<100 \mu \mathrm{m})$ with the mixing to mildly reacting $(\mathrm{M}-\mathrm{MR})$ fluid interface defined (MR fluid thickness $>$ $300 \mu \mathrm{m})$. The diagrams in Fig. 4 illustrate the multi-fluid interface probabilities for $\Phi$ $=0.4$ and 1.0. For $\Phi=0.4(\mathrm{Da}=0.14)$ the mixing fluid is primarily $(95 \%)$ adjacent to the reactants, i.e. $38 \%$ of all interfaces, see Table 2.

A distinct change of the fluid states adjacent to the reactants is evident for $\Phi=1.0$ $(\mathrm{Da}=5.1)$ with the mixing fluid reduced from $95 \%$ to $16 \%$ in favour of the mildly reacting $(6 \%)$, strongly reacting $(42 \%)$ and product $(36 \%)$ fluids. The interface statistics are accordingly altered. The mildly reacting fluid for $\Phi=0.4$ is primarily adjacent to the product fluid with a probability around $24 \%$, while the mixing mildly reacting fluid interface accounts for $6 \%$. The detachment of the mildly reacting fluid from the reactants by mixing and/or product fluid interlayers indicates the need for thermal support to initialise $\mathrm{OH}$ producing chemical activity for $\mathrm{Da} \leq 1.0$. The balance of fluid states sharing an interface with the mildly reacting fluid is strongly Da dependent, see Table 2.

The observed rates of strain, discussed below, prevent self-sustained burning for mixtures with $\Phi \leq 0.4$ and thus the strongly reacting fluid is only defined for $\Phi \geq 0.6$ $(\mathrm{Da} \geq 1.0)$. The probability of the strongly reacting fluid being adjacent to reactants decreases from $42 \%$ for $\Phi=1.0(\mathrm{Da}=5.1)$, to 31 for $\Phi=0.8(\mathrm{Da}=3.9)$ and $3 \%$ for $\Phi=0.6(\mathrm{Da}=1.2)$ as also shown in Table 2 by means of interface probabilities. 


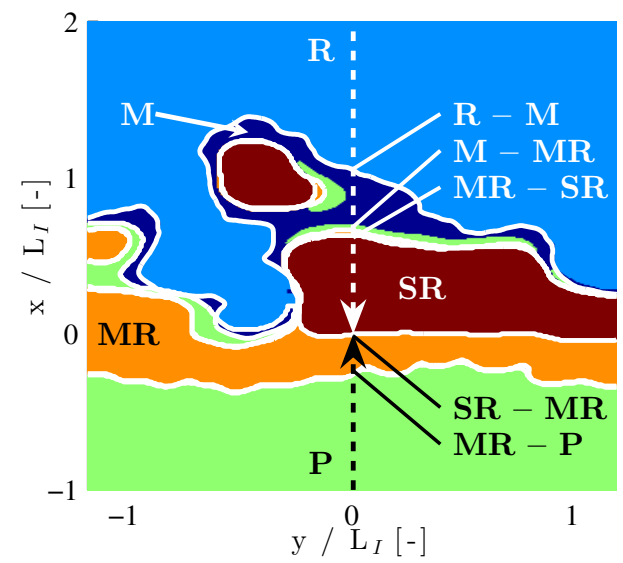

Figure 3: Instantaneous quinary multi-fluid field for DME/air at $\Phi=0.8$ truncated around the stagnation point. Vertical white/black arrows show the theoretical stagnation point streamline (SPS). Interfaces are defined by the intersection of the SPS and material surfaces (white iso-contours). R reactants (light blue field); $\mathrm{M}$ - mixing (dark blue); $\mathrm{MR}$ - mildly reacting (orange); SR - strongly reacting (red); $\mathrm{P}-$ products (green).
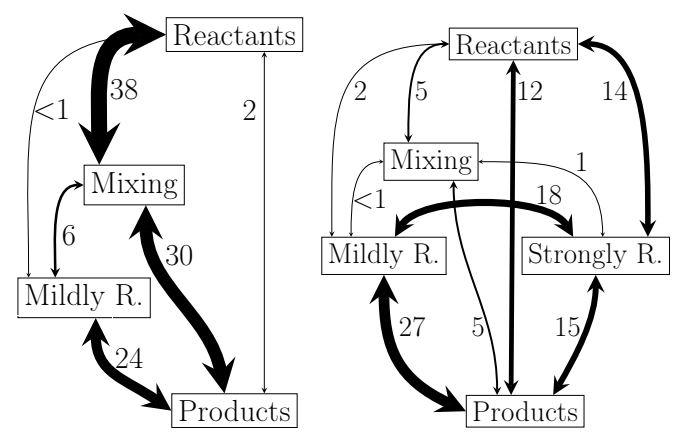

Figure 4: Multi-fluid interface diagram for $\mathrm{Da}=0.14(\Phi=0.4)$ (left) and $\mathrm{Da}=5.1(\Phi=1.0)$ (right) with all cases shown in Table 2. A distinct shift towards more reactive and exothermic intermediate fluids is illustrated by means of the probability weighted arrows indicating adjacent fluid states.

The interface of the mixing and strongly reacting fluid shows the reversed trend and dominates in the transitional case with $\mathrm{Da} \simeq 1.0(\Phi=0.6)$. The residual strongly reacting fluid shares interfaces with the mildly reacting or product fluids.

\subsection{Conditional Strain Distribution on Material Surfaces}

The in-plane velocity gradients were conditioned upon the fluid state material surfaces $(\beta)$. The strain rate $\left(e_{i j}=0.5\left(\partial u_{i} / \partial x_{j}+\partial u_{j} / \partial x_{i}\right)\right)$ and vorticity $\left(\omega_{i j}=\right.$ $\left.\partial u_{i} / \partial x_{j}-\partial u_{j} / \partial x_{i}\right)$ tensors were determined from the instantaneous planar PIV fields and subsequently conditioned upon $\beta$ : $e_{\beta, i j}=e_{i j} \mid \beta$ and $\omega_{\beta, i j}=\omega_{i j} \mid \beta$, where $\beta \equiv 1$ at the iso-contour trajectory and nil elsewhere. The tangent to the iso-contour was 
estimated at all positions $(\zeta)$ by a $3^{r d}$ order polynomial that was evaluated in the range of \pm 16 pixels along $\beta$ (twice the vector spacing). The strain rate tensor was rotated by the angle $\Theta(=\pi / 2-\theta)$ via $f_{\beta}=\mathbf{R} \cdot e_{\beta}$, where $\mathbf{R}$ is the rotation matrix and $\theta$ is the angle between the tangential on $\beta \mid \zeta$ and the SPS to define the normal $\left(a_{n}=f_{\beta, 11}\right)$ and tangential $\left(a_{t}=f_{\beta, 22}\right)$ strain components. The total strain was here defined as: $a_{d}=e_{\beta, 11}+e_{\beta, 22}$.

\subsubsection{Strain Distribution along the Reactant Fluid Surface}

The probability density function (PDF) of the $\mathrm{a}_{n}$ along the reactant fluid surface, depicted in Fig. 5, decreases progressively from mean compressive values found in the stagnation plane proximity $\left(-1550 \mathrm{~s}^{-1}\right)$ for $\mathrm{Da}<1.0$ to below the bulk strain rate based on the nozzle separation $\left(-440 \mathrm{~s}^{-1}\right)$ for Da $\simeq 5$. For high Da numbers, the $\operatorname{PDF}\left(a_{n}\right)$ is skewed towards extensive strains with its mode shifted from -1680 to $-330 \mathrm{~s}^{-1}$. This can be attributed to the rapid transition to chemically active fluids states and reaction onset away from the stagnation plane. The detachment causes a modest reduction of the mean tangential component $\left(950<a_{t}<690 \mathrm{~s}^{-1}\right)$ with a distinctly squeezed PDF spread (i.e. rms reduced by $\sim 30 \%$ ) and reversed mode shift $\left(550-690 \mathrm{~s}^{-1}\right)$ with increasing Da. The zero crossings, elevated skewness and reduced spread of the $\operatorname{PDF}\left(a_{n}\right)$ and $\operatorname{PDF}\left(a_{t}\right)$ with increasing Da has been observed in experiments [14] and DNS [18]. The impact of the detachment and adjacent flow acceleration for $\mathrm{Da}>1.0$ is shown by the total rate of strain in Fig. 5 (bottom left)

Table 2: Multi-fluid interface probabilities in percent by traversing along the stagnation point streamline in 3000 instantaneous images from reactants $(\mathrm{R})$ to products $(\mathrm{P})$. M - mixing fluid, MR - mildly reacting fluid, SR - strongly reacting fluid. Interfaces marked with $(-)$ are not defined.

\begin{tabular}{cc|ccccc}
\hline \multicolumn{2}{c|}{ Interface } & \multicolumn{3}{c}{$\Phi$} & & \\
& & & & \\
\multicolumn{2}{c}{ Samples } & 7466 & 7777 & 8802 & 9402 & 9762 \\
\hline $\mathrm{R}$ & $\mathrm{M}$ & 43 & 38 & 36 & 10 & 5 \\
$\mathrm{R}$ & $\mathrm{MR}$ & $<1$ & $<1$ & $<1$ & $<1$ & 2 \\
$\mathrm{R}$ & $\mathrm{SR}$ & - & - & 1 & 10 & 14 \\
$\mathrm{R}$ & $\mathrm{P}$ & 2 & 2 & 1 & 12 & 12 \\
$\mathrm{M}$ & $\mathrm{MR}$ & 1 & 6 & 8 & $<1$ & $<1$ \\
$\mathrm{M}$ & $\mathrm{SR}$ & - & - & 10 & 2 & 1 \\
$\mathrm{M}$ & $\mathrm{P}$ & 39 & 30 & 15 & 8 & 5 \\
$\mathrm{MR}$ & $\mathrm{SR}$ & - & - & 7 & 15 & 18 \\
$\mathrm{MR}$ & $\mathrm{P}$ & 15 & 24 & 21 & 25 & 27 \\
$\mathrm{SR}$ & $\mathrm{P}$ & - & - & 3 & 17 & 15 \\
\hline
\end{tabular}



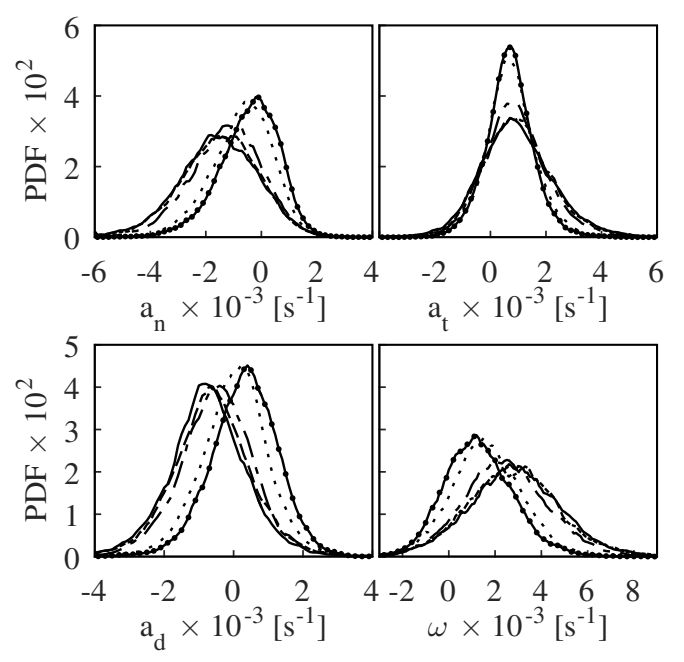

Figure 5: Rate of strain and vorticity evaluated along the reactant fluid surface: Top left: Normal strain; Right: Tangential strain; Bottom left: Total strain; Right Vorticity. Legend as Fig. 2.

with a shift from negative to positive mean values $\left(-610<a_{d}<250 \mathrm{~s}^{-1}\right)$. The earlier reaction onset further results in a reduced mean vorticity of the order of $50 \%$ for Da $=5.1$ compared to Da $<1.0$ (bottom right of Fig. 5). The Euclidean norm $(|a|=$ $\left.\left(a_{n}^{2}+a_{t}^{2}\right)^{1 / 2}\right)$ of the conditional strain along the reactant fluid material surface is listed in Table 1. The reaction onset of self-sustained flames is located in low strain regions $\left(|a|_{R}=1600 \mathrm{~s}^{-1}\right)$, while at low Da the iso-contour is found in high strain regions $\left(|a|_{R}\right.$ $\left.=2670 \mathrm{~s}^{-1}\right)$. The mixing fluid is found primarily adjacent to the reactant fluid with qualitatively the same trends and results are hence not shown.

\subsubsection{Strain Distribution along the Chemically Active Fluid Surfaces}

For $\mathrm{Da} \leq 1.0$, the mildly reacting fluid was spatially separated ( $>90 \%$ ) from the reactant fluid by a mixing and/or product fluid interlayer that suggests a need for thermal support. The strong impact of the HCP on the mildly reacting fluid surface results in a Da independent $a_{n} \simeq-1340 \mathrm{~s}^{-1}$ (see Fig. 6). The determined $a_{n}$ is well beyond the extinction strain rate (Table 1) for the transitional case with Da $\simeq 1.0\left(a_{q}=600 \mathrm{~s}^{-1}\right)$. The tails of the $\operatorname{PDF}\left(a_{n}\right)$ also exceed the extinction point for mixtures with $\mathrm{Da}>1.0\left(a_{q}=2000\right.$ and $3100 \mathrm{~s}^{-1}$ for $\Phi=0.8$ and 1.0). An increase in Da results in a modest tangential acceleration and elevated extensive tangential strain (top right in Fig. 6) with mean values progressively increasing (630, 710, 750, 
950, $990 \mathrm{~s}^{-1}$ for $\left.\Phi=0.2-1.0\right)$ and a reduced $\operatorname{PDF}$ spread. The shift of $\operatorname{PDF}\left(a_{d}\right)$ to decreasing compressive strain $(\sim 40 \%)$ with a constant spread is attributed to the increased dilatation [18]. The lack of flow alignment for low Da flows was also found by Chakraborty and Swaminathan $[18]$ at $\mathrm{Re}_{t} \simeq 50$. The turbulent mixing driven flow and importance of thermal HCP support for the mildly reacting fluid results in vorticity levels in the proximity of the stagnation plane (Fig. 6 bottom right) that are independent of Da.
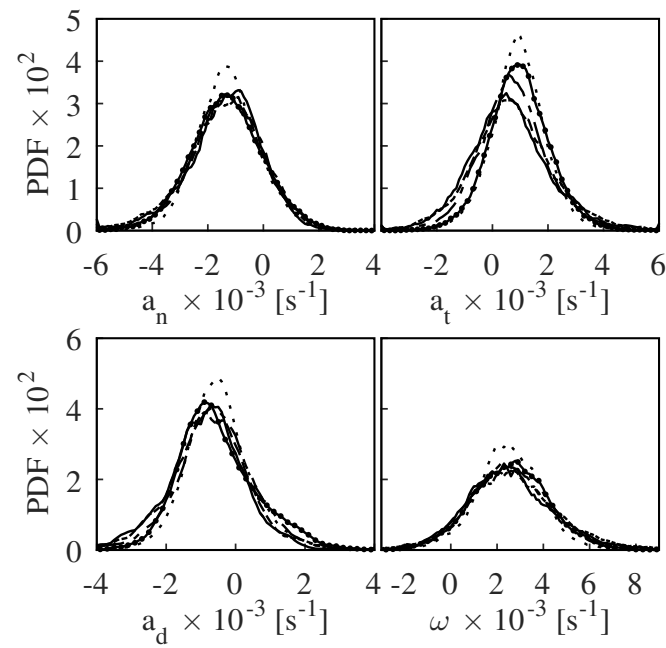

Figure 6: Rate of strain and vorticity evaluated along the mildly reacting fluid surface: Top left: Normal strain; Right: Tangential strain; Bottom left: Total strain; Right Vorticity. Legend as Fig. 2.

The transition to strongly exothermic fluid states collocates the flow acceleration normal to the flame front and results in a compressive strain reduction from -1050 to $-660 \mathrm{~s}^{-1}$, i.e. around a $40 \%$ reduction for $\Phi=1.0(\mathrm{Da}=5.1)$ compared to $\Phi$ $=0.6(\mathrm{Da}=1.2)$, as depicted in Fig. 7. This further corresponds to a decrease of $50 \%$ compared to the MR surface for $\Phi=1.0$. The tangential acceleration caused by combustion results in increased extensive strain $\left(650<a_{t}<830 \mathrm{~s}^{-1}\right)$. The shift of the $\operatorname{PDF}\left(a_{n}\right)$ towards reduced normal strain with a similar mean, but reduced spread of the $\operatorname{PDF}\left(a_{t}\right)$ coupled with a more pronounced skewness with increasing Da, was qualitatively also shown in DNS data by Chakraborty and Swaminathan [18]. A transition from contracting to dilating $a_{d}\left(-440\right.$ to $\left.100 \mathrm{~s}^{-1}\right)$ is evident for high Da as shown in the bottom left of Fig. 7. The increased dilatation with $\mathrm{Da}$ is further illustrated by comparing the strain along the reactant and strongly reacting fluid surface $(\Delta a=\mathrm{a} \mid \mathrm{R}$ 
- a|SR). For $\mathrm{Da} \simeq 1.0$, an increase of $\Delta a_{n}=-230 \mathrm{~s}^{-1}$ towards higher normal compressive strain and a reduced tangential strain $\left(\Delta a_{t}=-250 \mathrm{~s}^{-1}\right)$ is observed. By contrast, $a_{n}$ reduces by $\Delta a_{n}=220 \mathrm{~s}^{-1}$ towards extensive values for $\mathrm{Da}=5.1$ with an increased tangential strain $\left(\Delta a_{t}=80 \mathrm{~s}^{-1}\right)$. The preferential alignment of the flame front normal perpendicular to the extensive strain [17] becomes evident at high Da due to the favoured stabilisation of self-sustained flames in low compressive strain regions that are detached from the stagnation plane. Under such conditions, the strain acting on the strongly reacting material surface $\left(|a|_{S R}\right)$ is significantly lower than the extinction strain $\left(a_{q}\right)$, see Table 1 . The reduction in turbulent flame speed for lower Da [4] leads to a flame anchoring in the proximity of the stagnation plane with elevated normal strain levels and attenuated dilatation $[20]\left(|a|_{S R}>a_{q}\right.$ for Da $\left.\leq 1.2\right)$. The more ordered flow field with increasing $\mathrm{Da}$ is also reflected by a significant vorticity mitigation (bottom right in Fig. 7 ) of the order of $25 \%$ for $\Phi=1.0$ ( $\mathrm{Da}=5.1$ ) compared to $\Phi$ $=0.6(\mathrm{Da}=1.2)$. This also corresponds to a $\sim 40 \%$ reduction of the mean strongly reacting fluid vorticity compared to the mildly reacting value for $\Phi=1.0$.
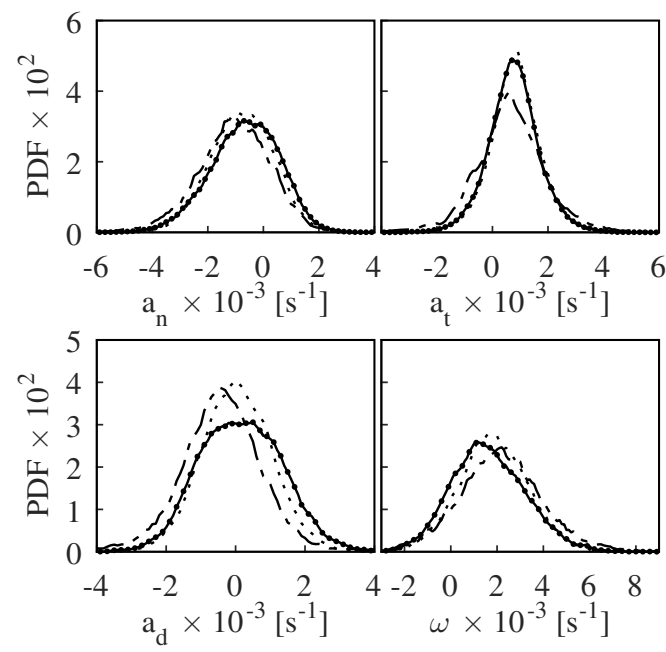

Figure 7: Rate of strain and vorticity evaluated along the strongly reacting fluid surface: Top left: Normal strain; Right: Tangential strain; Bottom left: Total strain; Right Vorticity. Legend as Fig. 2.

\subsection{Bimodal Flow Analysis}

Bimodal statistics are inferable from the multi-fluid approach by combining the thermally altered fluid states (i.e. mixing, mildly reacting, strongly reacting and prod- 


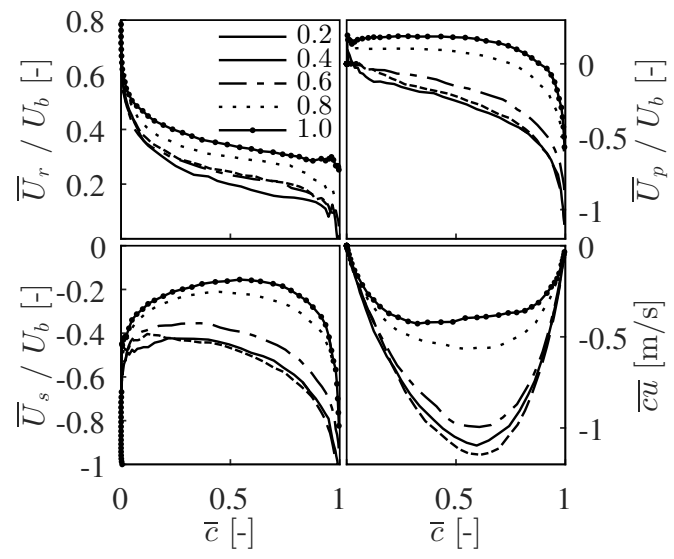

Figure 8: Mean conditional reactant ( $\bar{U}_{r}$, top left) and product ( $\bar{U}_{p}$, top right) velocities in progress variable $(\bar{c})$ space along with the slip velocity $\left(\bar{U}_{s}\right.$, bottom left) and scalar flux ( $\overline{c u}$, bottom right). The legend refers to $\Phi$.

uct fluid) to a bimodal product defining $\bar{c}[23]$. The probability of encountering reactant fluid is consequently $(1-\bar{c})$. The earlier reaction onset with increasing Da due to the higher turbulent burning velocity is illustrated by means of distinctly elevated mean axial reactant velocities $\left(\overline{U_{r}} / U_{b}\right)$ throughout the entire flame brush as shown in Fig. 8. The velocity of the lumped product fluids $\left(\overline{U_{p}} / U_{b}\right)$ illustrates a flame brush that is strongly affected by the counter-flowing $\mathrm{HCP}$ for $\mathrm{Da} \leq 1.0$. The quick transition to negative $\bar{U}_{p} / U_{b}$ indicate a $\mathrm{HCP}$ governed burning mode. Cases with $\mathrm{Da}>1.0$ exhibit a positive $\bar{U}_{p} / U_{b}$, i.e. are aligned with the natural reactant flow direction, up to $\bar{c}=$ 0.8 (0.9) for $\Phi=0.8(1.0)$. The corresponding slip velocity $\left(\bar{U}_{s} / U_{b}\right)$ shows the impact of increased dilatation with increasing Da as also illustrated by the scalar flux $(\overline{c u})$. Distinctly reduced gradient transport is shown in Fig. 8. However, transition to counter-gradient transport is suppressed even for the stoichiometric flame. By contrast, a transition from gradient to counter-gradient turbulent transport was shown for lean premixed twin $[4,27]$ and supported BTB flames [9] at $\operatorname{Re}_{t} \approx 120$.

\section{Conclusions}

The present work used a back-to-burnt opposed jet configurations to stabilise premixed DME/air flames against hot combustion products coupled with a multi-fluid approach to highlight the underlying causes leading to gradual combustion regime transitions from the corrugated flamelet regime towards distributed reactions for $\Phi$ 
$=0.2-1.0$. The analysis has provided new evidence on the link between Da and material surface strain at a constant $\operatorname{Re}_{t} \simeq 380$ with prominent characteristic changes in the burning mode observed. The fluid state that represents self-sustained burning was observed with a $40 \%$ probability directly adjacent to the reactant fluid for $\Phi=$ $1.0(\mathrm{Da}=5.1)$, which was significantly reduced with Da in favour of interlayers of reduced reactivity. The less reactive fluid states were also found to be increasingly separated from the reactants by a layer acting as an enthalpy source. This resulted in a mildly reacting fluid peak probability that was shifted towards the stagnation plane.

The proximity of the stagnation plane was characterised by a high compressive axial strain that increasingly prevented self-sustained burning for reduced Damköhler numbers. The increasing influence of hot combustion product support resulted in highly strained Da independent chemically active zones with no favoured dilatation direction - consistent with an auto-ignition related burning mode. By contrast, a preferential alignment of the flame front normal with the principal extensive strain and significantly reduced vorticity levels were evident for $\mathrm{Da}>1.0$. The associated dilatation caused a reduction of the gradient scalar flux. However, transition to a counter-gradient scalar transport was found to be suppressed under the current conditions $\left(\operatorname{Re}_{t} \simeq 380\right)$. The data are expected to be particularly valuable for establishing the range of validity of topologically based approaches and for the development of models that do not presume a flame front structure and thus applicable at lower Damköhler numbers.

\section{Acknowledgements}

The authors would like to acknowledge the support of the AFOSR and EOARD under Grant FA8655-13-1-3024 and thank Dr Chiping Li and Dr Gregg Abate for encouraging the work. The US Government is authorised to reproduce and distribute reprints for Governmental purpose notwithstanding any copyright notation thereon. The authors would also like to thank Dr Robert Barlow for his support.

\section{References}

[1] A. R. Masri, R. W. Dibble, R. S. Barlow, Prog. Energy Combust. Sci. 22 (1996) pp. 307-362. 
[2] R. S. Barlow, J. H. Frank, Proc. Comb. Inst. 27 (1998) pp. 1087-1095.

[3] D. Geyer, A. Kempf, A. Dreizler, J. Janicka, Combust. Flame 143 (2005), pp. 524-548.

[4] K. H. H. Goh, P. Geipel, R. P. Lindstedt, Combust. Flame 161 (2014), pp. 2419-2434.

[5] J. A. Wünning, J. G. Wünning, Prog. Energ. Combust. 23 (1997), pp. 81-94.

[6] T. Plessing, N. Peters, J. G. Wünning, Proc. Combust. Inst. 27 (1998), pp. 3197-3204.

[7] F. J. Weinberg, Nature 233 (1971), pp. 239-241.

[8] E. Mastorakos, A. Taylor, J. Whitelaw, Combust. Flame 102 (1995), pp. 101-114.

[9] K. H. H. Goh, P. Geipel, F. Hampp, R. P. Lindstedt, Proc. Combust. Inst. 34, (2013), pp. 33113318.

[10] K. H. H. Goh, PhD thesis, Imperial College, Aug 2013, url: http://hdl.handle.net/10044/1/28073.

[11] F. Hampp, R. P. Lindstedt, In Y. Sakai and C. Vassilicos (eds.) Fractal Flow Design: How to Design Bespoke Turbulence and why, Springer-Verlag, CISM Int. Mech. Sci. 568, 2016, DOI:10.1007/978-3-319-33310-6 3 .

[12] B. Coriton, J. H. Frank, A. Gomez, Combust. Flame 160 (2013), pp. 2442-2456.

[13] B. Böhm, C. Heeger, I. Boxx, W. Meier, A. Dreizler, Proc. Combust. Inst. 32 (2009), pp. 16471654.

[14] G. Hartung, J. Hult, C. F. Kaminski, J. W. Rogerson, N. Swaminathan, Phys. Fluids 20 (2008), 035110 .

[15] A. M. Steinberg, J. F. Driscoll, Combust. Flame 156 (2009), pp. 2285-2306.

[16] A. M. Steinberg, J. F. Driscoll, N. Swaminathan, Combust. Flame 159 (2012), pp. 2576-2588.

[17] A. M. Steinberg, B. Coriton, J. H. Frank, Proc. Combust. Inst. 35 (2015), pp. $1287-1294$.

[18] N. Chakraborty, N. Swaminathan, Phys. Fluids 19 (2007), 045103.

[19] L. Wang, N. Chakraborty, J. Zhang, Proc. Combust. Inst. 34 (2013), pp. 1401-1409.

[20] Y. Gao, N. Chakraborty, N. Swaminathan, J. Combust. (2014), 280671.

[21] D.B. Spalding, Proc. $7^{\text {th }}$ Biennial Conf. on Comp. Techn. Applications (CTAC 95), World Scientific Publishing Co Ltd, (1996) pp. 59-81.

[22] F. Hampp, PhD thesis, Imperial College, May 2016, url: http://hdl.handle.net/10044/1/32582.

[23] K. N. C. Bray, in N. Swaminathan, K. N. C. Bray (Eds.), Turbulent Premixed Flames, Cambridge University Press, 2011, pp. 41-60.

[24] C. Arcoumanis, C. Bae, R. Crookes, E. Kinoshita, Fuel 87 (2008), pp. 1014-1030.

[25] S.-W. Park, PhD thesis, Imperial College, March 2012, url: http://hdl.handle.net/10044/1/9599.

[26] K. H. H. Goh, P. Geipel, F. Hampp, R. P. Lindstedt, Fluid Dyn. Res. 45, (2013), 061403.

[27] K. H. H. Goh, P. Geipel, R. P. Lindstedt, Proc. Combust. Inst. 35 (2015), pp. 1469-1476.

[28] P. Geipel, K. H. H. Goh, R. P. Lindstedt, Flow Turbul. Combust. 85 (2010) pp. 397-419.

[29] W. P. Jones, R. P. Lindstedt, Combust. Sci. Techn. 61 (1988), pp. 31-49.

[30] L. W. Kostiuk, K. N. C. Bray, T. C. Chew, Combust. Sci. Technol. 64 (4-6) (1989) pp. $233-241$.

[31] W. K. George, H. J. Hussein, J. Fluid Mech. 233 (1991), pp. 1-23.

[32] B. Coriton, M. D. Smooke, A. Gomez, Combust. Flame 157 (2010), pp. 2155-2164. 
[33] J. Kerl, T. Sponfeldner, F. Beyrau, Combust. Flame 158 (2011), pp. 1905-1907.

[34] M. Raffel, C. E. Willert, S. Wereley, J. Kompenhans, Springer-Verlag, 2007, ISBN: 978-3-64243166-1.

[35] D. Han, M. G. Mungal, Combust. Flame 132 (2003), pp. 565-590.

[36] B. Wieneke, K. Pfeiffer, $15^{\text {th }}$ Int. Symp. Appl. Laser Tech. Fluid Mech. (2010) url: http://ltces.dem.ist.utl.pt/LXLASER/lxlaser2010/upload/1845_qkuqls_1.12.3.Full_1845.pdf.

[37] Y. Zhu, R. A. Antonia, J. Fluid Mech. 287 (1995) pp. 199-223.

[38] T. Zhou, R. A. Antonia, J-J. Lasserre, M. Coantic, F. Anselmet, Exp. Fluids 34 (2003) pp. $449-$ 459 .

[39] P. Lavoie, G. Avallone, F. de Gregorio, G. P. Romano, R. A. Antonia, Exp. Fluids 43 (2007) pp. 39-51.

[40] K. Nishino, M. Samada, K. Kasuya, K. Torii, Int. J. Heat Fluid Fl. 17 (1996) pp. $193-201$.

[41] S. K. Sinha, Exp. Fluids 6 (1988) pp. 67-68.

[42] I. G. Shepherd, R. K. Cheng, P. J. Goix, Proc. Combust. Inst. 23 (1991), pp. $781-787$.

[43] A. M. Steinberg, J. F. Driscoll, S. L. Ceccio, Exp. Fluids 44 (2008), pp. 985-999.

[44] Photographic Lenses, Tech. Rep. MIL-STD-150A, (1963).

[45] K. A. Buch, W. J. A. Dahm, J. Fluid Mech. 364 (1998) pp. 1-29.

[46] G. K. Batchelor, J. Fluid Mech. 5 (1959) pp. 113-133.

[47] N. Peters, J. Fluid Mech. 384 (1999) pp. 107-132.

[48] C. J. Lawn, R. W. Schefer, Combust. Flame 146 (2006) pp. 180-199. 
3661 Overview of experimental conditions. The extinction strain rates $a_{q}\left(s^{-1}\right)$ were computed in the corresponding twin flame configuration [22]. The Euclidean norm $\left(|a|=\left(a_{n}^{2}+a_{t}^{2}\right)^{1 / 2}\right)$ was evaluated along the reactant (R) and strongly reacting (SR) fluid surfaces. The integral length scale $\left(L_{I}=4.1 \mathrm{~mm}\right)$ and velocity fluctuation $\left(\mathrm{u}^{\prime}=1.6 \pm 0.04 \mathrm{~m} \mathrm{~s}^{-1}\right)$ were

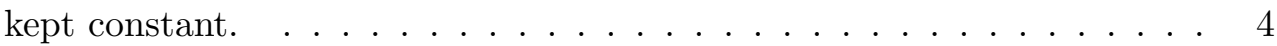

2 Multi-fluid interface probabilities in percent by traversing along the stagnation point streamline in 3000 instantaneous images from reactants $(\mathrm{R})$ to products $(\mathrm{P})$. M - mixing fluid, $\mathrm{MR}$ - mildly reacting fluid, SR - strongly reacting fluid. Interfaces marked with $(-)$ are not defined. 10 


\section{List of Figures}

1 Schematic of the experimental configuration with the coordinate system convention indicated. SP - stagnation plane; DSI - density segregation iso-contour; $\mathrm{HCP}$ - hot combustion products; CFG - cross fractal grid; FSM - flame stabilising mesh; FBA - flash back arrestor. . . . . . . . . 3

2 Multi-fluid probability statistics in physical space along the stagnation point streamline and aligned at the $\bar{c}=0.02$ iso-contour. Top left: Reactant (black) and product fluid probability (blue/grey); Right: Mixing fluid probability $\times 4$; Bottom left: Mildly reacting fluid probability $\times$ 4; Right: Strongly reacting fluid probability $\times 2$. Legend entries refer to the equivalence ratio with the corresponding Da listed in Table 1. . . 7

3 Instantaneous quinary multi-fluid field for DME/air at $\Phi=0.8$ truncated around the stagnation point. Vertical white/black arrows show the theoretical stagnation point streamline (SPS). Interfaces are defined by the intersection of the SPS and material surfaces (white iso-contours). $\mathrm{R}$ - reactants (light blue field); $\mathrm{M}$ - mixing (dark blue); $\mathrm{MR}$ - mildly reacting (orange); $\mathrm{SR}$ - strongly reacting (red); $\mathrm{P}$ - products (green). . 9

4 Multi-fluid interface diagram for $\mathrm{Da}=0.14(\Phi=0.4)$ (left) and $\mathrm{Da}=$ $5.1(\Phi=1.0)$ (right) with all cases shown in Table 2. A distinct shift towards more reactive and exothermic intermediate fluids is illustrated by means of the probability weighted arrows indicating adjacent fluid

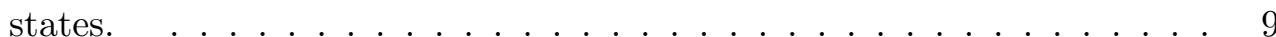

5 Rate of strain and vorticity evaluated along the reactant fluid surface: Top left: Normal strain; Right: Tangential strain; Bottom left: Total strain; Right Vorticity. Legend as Fig. 2. . . . . . . . . . . . . . . 11

6 Rate of strain and vorticity evaluated along the mildly reacting fluid surface: Top left: Normal strain; Right: Tangential strain; Bottom left: Total strain; Right Vorticity. Legend as Fig. 2. . . . . . . . . . . . . . . 12 
$7 \quad$ Rate of strain and vorticity evaluated along the strongly reacting fluid surface: Top left: Normal strain; Right: Tangential strain; Bottom left:

8 Mean conditional reactant $\left(\bar{U}_{r}\right.$, top left) and product ( $\bar{U}_{p}$, top right) velocities in progress variable $(\bar{c})$ space along with the slip velocity $\left(\bar{U}_{s}\right.$, bottom left) and scalar flux ( $\overline{c u}$, bottom right). The legend refers to $\Phi$. 